\title{
Piezoelectric-field-enhanced lateral ambipolar diffusion coefficient in InGaN/GaN multiple quantum wells
}

\author{
Yin-Chieh Huang, Jian-Chin Liang, and Chi-Kuang Sun ${ }^{\text {a) }}$ \\ Department of Electrical Engineering and Graduate Institute of Electro-Optical Engineering, National \\ Taiwan University, Taipei 10617, Taiwan, Republic of China \\ Amber Abare and Steven P. DenBaars \\ Department of Electrical and Computer Engineering, University of California, Santa Barbara, \\ California 93106
}

(Received 10 October 2000; accepted for publication 14 December 2000)

\begin{abstract}
Lateral diffusion behavior of two-dimensional carrier gas in InGaN/GaN multiple quantum wells was investigated using optical transient transmission measurements. A large ambipolar diffusion coefficient was observed, which was attributed to the enhancement by a strong piezoelectric field. This large ambipolar diffusion coefficient was found to increase with increased well width with a value on the order of $3000 \mathrm{~cm}^{2} / \mathrm{s}$ for a $62 \AA$ well-width sample. (C) 2001 American Institute of Physics. [DOI: 10.1063/1.1347399]
\end{abstract}

During the past few years development in the field of GaN-based semiconductors has received considerable interest because of its applications as light emitters ${ }^{1,2}$ and light detectors ${ }^{3,4}$ in the green to UV wavelength range as well as high power and high temperature electronics. ${ }^{5,6}$ Understanding the transport properties in $\mathrm{GaN}$ material systems is fundamental to improving device performance. Monte Carlo calculations predicted a high peak velocity, high saturation velocity, and relatively high electron mobility in $\mathrm{GaN}^{7}$ and InN. ${ }^{8}$ Recent extensive studies ${ }^{9-14}$ on the two-dimensional (2D) electron gas at the $\mathrm{AlGaN} / \mathrm{GaN}$ heterointerface indicated large 2D electron mobility, which is much higher than that for bulk GaN. Low temperature mobility values of over $10000 \mathrm{~cm}^{2} / \mathrm{V}$ s were reported. ${ }^{12-14}$ Connected with mobility through the Einstein relation, the diffusion coefficient in bulk GaN was also extensively studied. ${ }^{15-17}$ Electron and hole diffusion coefficient values on the order of $0.1-10 \mathrm{~cm}^{2} / \mathrm{s}$ were reported. ${ }^{15-18}$ Studies of 2D lateral diffusion in $\mathrm{GaN}$ related structures were, on the other hand, rarely reported.

In this letter, we report our studies on the 2D lateral diffusion behavior in InGaN/GaN multiple quantum wells (MQWs) using an optical transient transmission measurement technique. The 2D ambipolar diffusion coefficient, which describes the excess electron-hole pair diffusion ${ }^{19}$ in a 2D plane, was measured. Similar to previous observation of the giant ambipolar diffusion coefficient in GaAs based $n-i-p-i$ superlattices ${ }^{20}$ where fast in-plane diffusion was greatly enhanced by the spatial separation of electrons and holes due to strong built-in electric fields normal to the layers, we have observed an enhanced ambipolar diffusion coefficient in large well-width InGaN MQWs due to the spatial charge separation by the large built-in piezoelectric field. With a well width increased from 12 to $62 \AA$, the measured ambipolar diffusion coefficient at room temperature was found to increase from $400 \pm 100 \mathrm{~cm}^{2} / \mathrm{s}$ up to 2700 $\pm 500 \mathrm{~cm}^{2} / \mathrm{s}$.

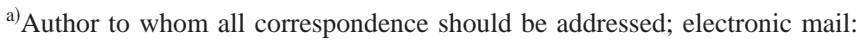
sun@cc.ee.ntu.edu.tw
}

The InGaN/GaN MQWs we studied were grown on $c$-plane sapphire using atmospheric pressure metalorganic chemical vapor deposition. After the deposition of a $\mathrm{GaN}$ nucleation layer, the temperature was raised to $1080^{\circ} \mathrm{C}$ to grow $n$-type $\mathrm{GaN}$ of $2.5 \mu \mathrm{m}$ thickness. The 14 period InGaN/GaN MQWs were then grown on this thick GaN layer at a temperature of $790^{\circ} \mathrm{C}$. The InGaN wells were unintentionally doped with a $n$-type carrier density less than 1 $\times 10^{17} \mathrm{~cm}^{-3}$, determined from Hall measurement of a calibration sample grown on the same condition. The barriers were Si doped ( $n$ type) with a Si density on the order of 2 $\times 10^{18} \mathrm{~cm}^{-3}$, determined by secondary ion mass spectrometry on a calibration sample grown under the same condition. The sample was finished with a $0.1 \mu \mathrm{m}$ cap $\mathrm{Al}_{0.1} \mathrm{Ga}_{0.9} \mathrm{~N}$ layer. The barrier widths of all samples were held at $43 \AA$ while the well widths were varied from 12 to $62 \AA$ (with 12 , 25,50 , and $62 \AA$ ). The crystal structure was wurzite. The In composition in the MQWs was around $10 \%$. The band gaps of MQWs were between $390-430 \mathrm{~nm}$ for $12-62 \AA$ wellwidth MQWs. The piezoelectric field inside the InGaN QW was estimated to be around $350 \mathrm{kV} / \mathrm{cm}^{21}$ Time-resolved photoluminescence studies on these samples revealed a carrier lifetime between 1-4 ns for short well-width samples (12 and $25 \AA$ ) and a much longer lifetime of $>35$ ns for the large well-width samples (50 and $62 \AA$ ), due to the electron/ hole wave function separation. ${ }^{21}$

The experiments were performed using a standard transmission pump-probe technique ${ }^{22}$ with a Kerr-lens-mode locked Ti:sapphire laser. The laser output pulses from the femtosecond Ti:sapphire laser were frequency doubled in a BBO crystal to reach the band gap energy of the InGaN/GaN MQWs. The frequency-doubled pulses had a pulsewidth of $180 \mathrm{fs}$ at a wavelength of $390 \mathrm{~nm}$. One tenth of the UV beam was reflected by a beamsplitter to be used as the probe beam while the rest of the UV power passed through the beamsplitter was used as the pump beam. The pump and probe beams were focused vertically onto the InGaN/GaN MQW sample by an objective lens. The resulted signal of the probe 
beam was then averaged and recorded as a function of the temporal delay between the pump and probe beams.

With a fundamental transmission electron microscope Gaussian mode, the UV pump pulses excited carriers inside the wells with a Gaussian-like spatial density distribution. Due to the quantum-confinement effect inside the wells, the photoexcited nonuniform carriers would diffuse laterally out of the excitation pump region but would still be confined inside each of the quantum wells. Using a delayed probe pulse with the same spot size as the pump pulse, its transmission variation would then reflect the carrier density change in the pumping region that was governed by the $2 \mathrm{D}$ lateral diffusion process. The 2D lateral diffusion process obeys the diffusion equation

$$
\frac{\partial N}{\partial t}=D \nabla^{2} N=D\left[\frac{1}{r} \frac{\partial}{\partial r}\left(r \frac{\partial}{\partial r} N\right)\right],
$$

where $N$ is the excited carrier population, $t$ is time, $D$ is the diffusion coefficient, and $r$ is the distance from the pump beam spot center in the diffusion plane. The general solution of this $2 \mathrm{D}$ diffusion equation is

$$
N(r, t)=\frac{C_{1}}{t} e^{\left(-r^{2} / 4 D t\right)},
$$

where $C_{1}$ is a constant. With an initial Gaussian distribution of $N\left(r, t_{0}\right)=C_{2} / w^{2} e^{-2 r^{2} / w^{2}}$, where $w$ is the pump beam radius and $C_{2}$ is a constant, the initial diffusion time constant $\tau\left(t_{0}\right)$ at a distance $r$ away from the pump beam center can be described as

$$
\tau(r)=\frac{1}{\frac{8 D}{w^{2}}-\frac{16 D r^{2}}{w^{4}}} .
$$

With Eq. (3), we found that $\tau(r=0)=w^{2} / 8 D$. However, with a probe spot size equal to the pump spot size, the initial "effective" diffusion time constant probed by the whole probe laser beam would be modified as

$$
\tau_{\text {effective }}=\frac{w^{2}}{4 D}
$$

by integrating the diffusion effect throughout the 2D beam profile. A square relation between the initial effective diffusion time constant and laser spot size was found. By measuring the diffusion time constant as a function of beam spot size, the values of 2D lateral diffusion coefficient $D$ can then be obtained.

Figure 1 shows the room-temperature measured transient transmission responses in the $12 \AA \mathrm{InGaN} / \mathrm{GaN} \mathrm{MQW}$ at a wavelength of $390 \mathrm{~nm}$ corresponding to different laser beam radii from 4.8 to $11 \mu \mathrm{m}$ on the sample. The average pumpinduced excess photocarrier density was estimated to be on the order of $0.3-3 \times 10^{12} \mathrm{~cm}^{-2}$. With a fixed pump power and by varying the laser spot size on the sample, we not only observed a transmission response decrease due to reduced average photocarrier densities, the time constant for the transient transmission change was also found to increase with increased spot size. We have also performed similar measurements by using a fixed spot size but varying the optical pump power, no significant time constant variation was ob-

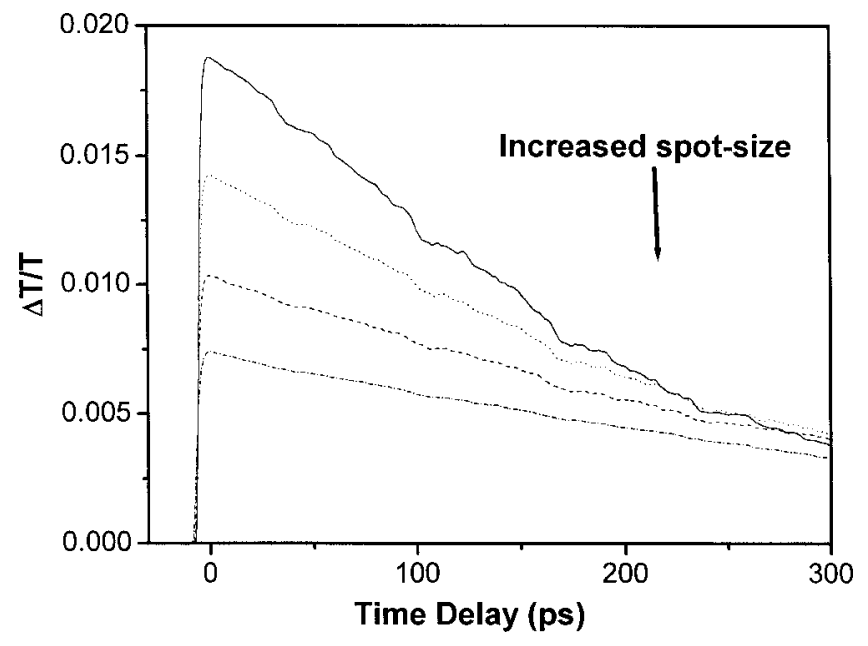

FIG. 1. Transient response of the $12 \AA$ InGaN/GaN MQW with different spot sizes. The spot size was increased from 4.8 to $11 \mu \mathrm{m}$. The laser excitation wavelength was $390 \mathrm{~nm}$.

served, which is different from the size-variation experiments. The time constant was also found to be independent of the laser excitation wavelength. Figure 2 plots the measured time constant around time zero as a function of the pump spot size for the $12 \AA$ well-width MQW. The measured initial time constant was found to increase from 150 to $700 \mathrm{ps}$ when the spot size was increased from 4.8 to $11 \mu \mathrm{m}$. A square relationship between the initial time constant and the laser spot size can be found, agreeing with Eq. (4). By fitting Fig. 2 with Eq. (4), as shown in the dotted line, a large value of $400 \pm 100 \mathrm{~cm}^{2} / \mathrm{s}$ could be obtained for the $2 \mathrm{D}$ lateral diffusion coefficient $D$.

Similar measurements were also repeated in samples with different well widths. Figure 3 shows the measured transient transmission traces from different MQW samples with a fixed spot size of $11 \mu \mathrm{m}$. A drastic time constant decrease was observed with increased well width. With a fixed spot size of $11 \mu \mathrm{m}$, the measured diffusion time constant decreased from 700 to 100 ps when the sample well width increased from 12 to $62 \AA$. By performing beam radius dependent measurements, 2D lateral diffusion coefficient $D$ was found to increase drastically from $400 \pm 100$ to 2700

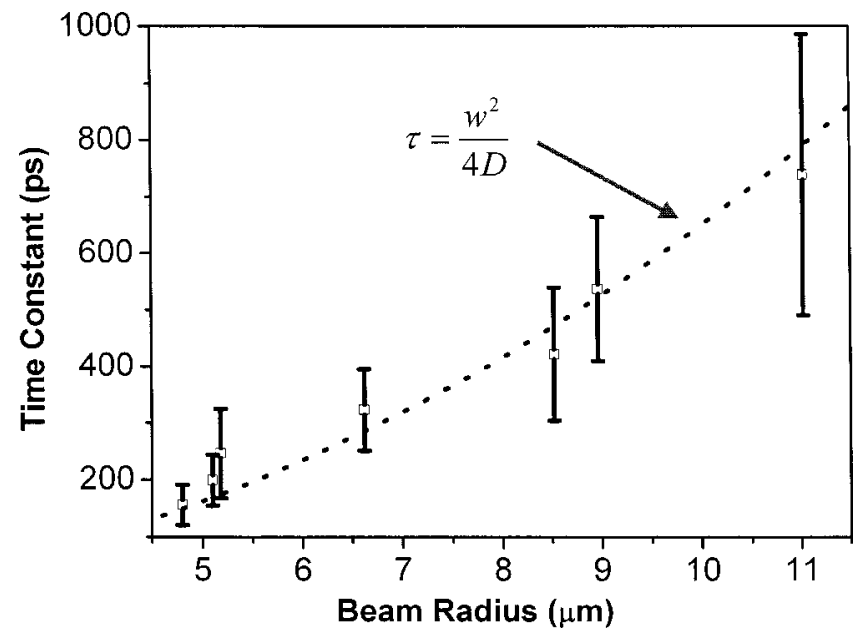

FIG. 2. The measured initial diffusion time constant vs pump beam radius for the $12 \AA$ well-width sample. The dotted line is a fitting according to Eq. (4) with a value of $D$ of $400 \mathrm{~cm}^{2} / \mathrm{s}$. 


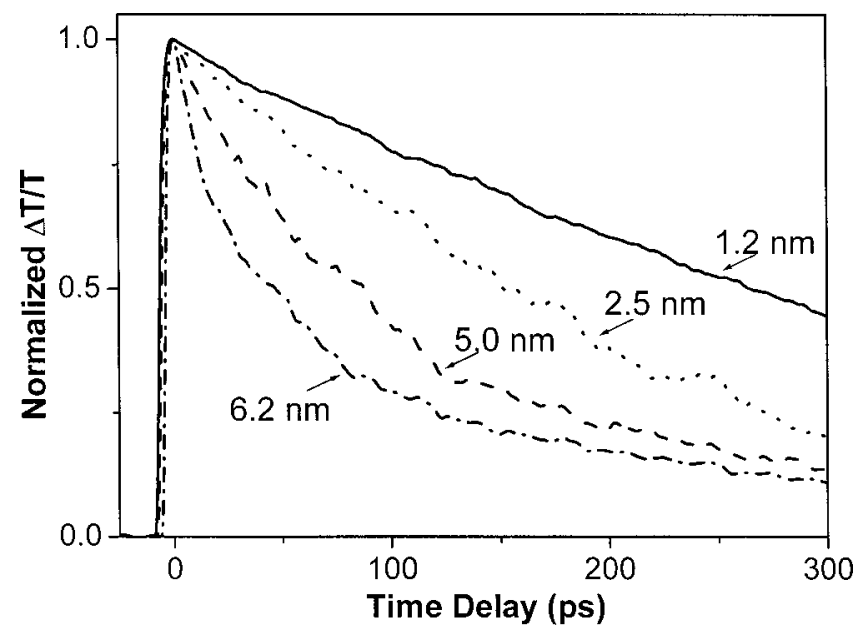

FIG. 3. The measured probe transient transmission change for different sample well widths. The laser excitation wavelength was $390 \mathrm{~nm}$ with a fixed spot size of $11 \mu \mathrm{m}$ on the measured sample.

$\pm 500 \mathrm{~cm}^{2} / \mathrm{s}$ when the sample well width increased from 12 to $62 \AA$. Figure 4 shows this measured result.

This interesting behavior is similar to the "giant ambipolar diffusion coefficient" previously observed in GaAs/ InGaAs $n-i-p-i$ or $p-i-n$ structures, ${ }^{20,23}$ where a diffusion coefficient exceeded $16000 \mathrm{~cm}^{2} / \mathrm{s}$ was reported. ${ }^{20}$ The observed ambipolar diffusion coefficients exceeded typical bulk value by several orders of magnitude. The fast $2 \mathrm{D}$ lateral diffusion in the previous observed systems was due to the spatial separation of the charge carriers of opposite sign which resulted from the strong built-in electric fields normal to the layers. ${ }^{20,23}$ Due to the decomposition of the electronhole plasma into its components, the attractive Coulomb interaction between electrons and holes was reduced and did no longer compensate the repulsion between carriers of the same type as it did in bulk materials. ${ }^{20,23}$ In addition, the electron-hole recombination was effectively suppressed by the spatial separation of the carriers. ${ }^{20,23}$ For the InGaN MQWs, the strong built-in piezoelectric field separates the generated carriers with opposite charges. This spatial charge separation inside the QW would be enhanced with increased well width and would be responsible for the observation of

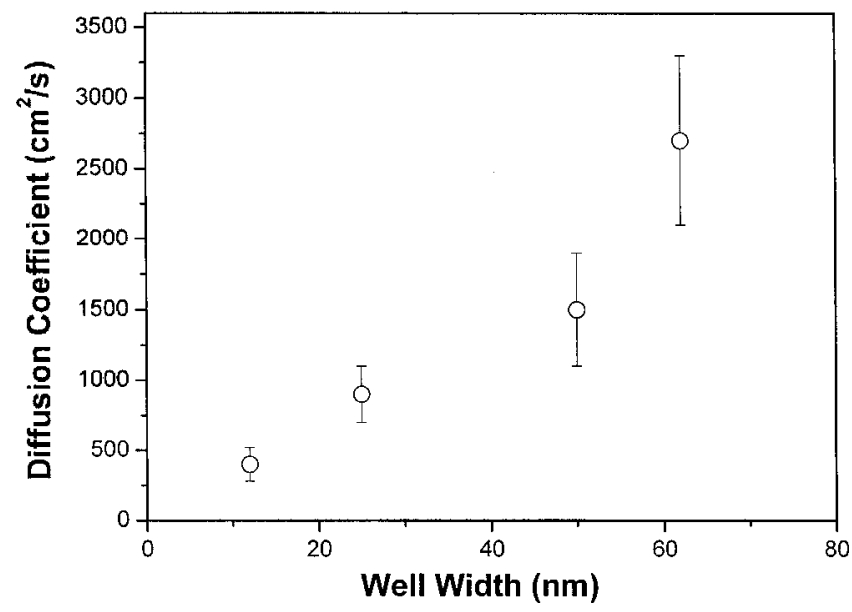

FIG. 4. Measured 2D diffusion coefficient vs InGaN MQW well width. the increased 2D lateral diffusion coefficient. The space charge separation inside the InGaN QW of thick well-width samples was also supported by previous TRPL measurements in the same samples. ${ }^{21}$

In conclusion, we studied the 2D lateral diffusion behaviors in InGaN/GaN MQWs using an optical transient transmission measurement technique. A large ambipolar diffusion coefficient was observed at room temperature. With a well width increased from 12 to $62 \AA$, the measured ambipolar diffusion coefficient was found to increase from $400 \pm 100$ to $2700 \pm 500 \mathrm{~cm}^{2} / \mathrm{s}$. Similar to previous observations of the giant ambipolar diffusion coefficient in $\mathrm{InGaAs} / \mathrm{GaAs}$ based $n-i-p-i$ or $p-i-n$ structures,$^{20,23}$ the observation of the enhancement of 2D lateral diffusion coefficient in InGaN MQWs with increased well width could be understood through the spatial separation of electrons and holes due to the strong built-in piezoelectric field inside the MQWs.

This work is sponsored by National Science Council of Taiwan, Republic of China under Grant No. NSC 89-2112M-002-082.

${ }^{1}$ S. Nakamura, M. Senoh, S. I. Nagahama, N. Iwasa, and T. Yamada, Jpn. J. Appl. Phys., Part 1 35, 74 (1996).

${ }^{2}$ S. Nakamura, Science 281, 956 (1998).

${ }^{3}$ M. Asif Khan, J. N. Kuznia, D. T. Olson, J. M. V. Hove, M. Blasingame, and L. F. Reitz, Appl. Phys. Lett. 60, 2917 (1992).

${ }^{4}$ X. Zhang, P. Kung, D. Walker, J. Piotrowski, A. Rogalski, A. Saxler, and M. Razeghi, Appl. Phys. Lett. 67, 2028 (1995).

${ }^{5}$ M. A. Khan, A. Bhattarai, J. N. Kuznia, and D. T. Olson, Appl. Phys. Lett. 63, 1214 (1993).

${ }^{6}$ U. K. Mishra, Y. F. Wu, B. P. Keller, S. Keller, and S. P. DenBaars, IEEE Trans. Microwave Theory Tech. 46, 756 (1998).

${ }^{7}$ U. V. Bhapkar and M. S. Shur, J. Appl. Phys. 82, 1649 (1997).

${ }^{8}$ S. K. O'Leary, B. E. Foutz, M. S. Shur, U. V. Bhapkar, and L. F. Eastman, J. Appl. Phys. 83, 826 (1998).

${ }^{9}$ M. Asif Khan, Q. Chen, C. J. Sun, M. Shur, and B. Gelmont, Appl. Phys. Lett. 67, 1429 (1995).

${ }^{10}$ C. F. Lin, H. C. Cheng, and J. A. Huang, M. S. Feng, J. D. Guo, and G. C. Chi, Appl. Phys. Lett. 70, 2583 (1997).

${ }^{11}$ R. Gaska, J. W. Yang, A. Osinsky, Q. Chen, M. Asif Khan, A. O. Orlov, G. L. Snider, and M. S. Shur, Appl. Phys. Lett. 72, 707 (1998).

${ }^{12}$ C. R. Elsass, I. P. Smorchkova, B. Heying, E. Haus, P. Fini, K. Maranowasi, J. P. Ibbetson, S. Keller, P. M. Petroff, S. P. DenBaars, U. K. Mishra, and J. S. Speck, Appl. Phys. Lett. 74, 3528 (1999).

${ }^{13}$ T. Wang, Y. Ohno, M. Jachab, D. Nakagawa, T. Shirahama, S. Sakai, and H. Ohno, Appl. Phys. Lett. 74, 3531 (1999).

${ }^{14}$ L. K. Li, B. Turk, W. I. Wang, S. Syed, D. Simonian, and H. L. Stormer, Appl. Phys. Lett. 76, 742 (2000).

${ }^{15}$ L. Chernyak, A. Osinsky, H. Temkin, J. W. Yang, Q. Chen, and M. Asif Khan, Appl. Phys. Lett. 69, 2531 (1996).

${ }^{16}$ J. Y. Duboz, F. Binet, D. Dolfi, N. Laurent, F. Scholz, J. Off, A. Sohmer, O. Briot, and B. Gil, Mater. Sci. Eng., B 50, 289 (1997).

${ }^{17}$ Z. Z. Bandic, P. M. Bridger, E. C. Piquette, and T. C. McGill, Appl. Phys. Lett. 73, 3276 (1998).

${ }^{18}$ H. Haag, B. Hönerlage, O. Briot, and R. L. Aulombard, Phys. Rev. B 60, 11624 (1999).

${ }^{19}$ S. M. Sze, Physics of Semiconductor Devices, 2nd Ed. (Wiley, New York, 1987), p. 87.

${ }^{20}$ K. H. Gulden, H. Lin, P. Kiesel, P. Riel, G. H. Döhler, and K. J. Ebeling, Phys. Rev. Lett. 66, 373 (1991).

${ }^{21}$ S. F. Chichibu, A. C. Abare, M. P. Mack, M. S. Minsky, T. Deguchi, D. Cohen, P. Kozodoy, S. B. Fleischer, S. Keller, J. S. Speck, J. E. Bowers, E. Hu, U. K. Mishra, L. A. Coldren, S. P. DenBaars, K. Wada, T. Sota, and S. Nakamura, Mater. Sci. Eng., B 59, 298 (1999).

${ }^{22}$ J.-C. Diels and W. Rudolph, Ultrashort Laser Pulse Phenomena (Academic, New York, 1996), pp. 401-411.

${ }^{23}$ D. Streb, G. Klem, W. Fix, P. Kiesel, and G. H. Döhler, Appl. Phys. Lett. 71, 1501 (1997). 